\title{
ON ECONOMIC OPTIMALITY OF MODEL PREDICTIVE CONTROL
}

\author{
A. Ferramosca ${ }^{*, 1}$ A. H. González ${ }^{*, 1}$ D. Limon ${ }^{* *, 3}$ \\ G. A. Bustos ${ }^{*, 2}$ J. L. Godoy ${ }^{*, 2}$ J. L. Marchetti ${ }^{* 1}$ \\ * Institute of Technological Development for the Chemical \\ Industry (INTEC), CONICET-Universidad Nacional del Litoral \\ (UNL). Güemes 3450, (3000) Santa Fe, Argentina - \\ \{ferramosca,alejgon,gbustos,jlgodoy,jlmarch\}@santafe- \\ conicet.gov.ar \\ ** Departamento de Ingeniería de Sistemas y Automática, \\ Universidad de Sevilla, Escuela Superior de Ingenieros, Camino \\ de los Descubrimientos s/n.41092,Sevilla,Spain -dlm@us.es.
}

\begin{abstract}
Resumen: Model Predictive Control (MPC) is the most used advanced control strategy in the industries, mainly due to its capability to fulfill economic objectives, taking into account a dynamic simplified model of the plant, constraints, and stability requirements. In the last years, several economic formulations of MPC have been presented, which get over the standard setpoint-tracking formulation. The goal of this work is to provide, by means of application to a highly nonlinear plant, a comparison of different strategies, focusing mainly on economic optimality, computational burden, and economic performance.
\end{abstract}

Palabras Claves: Model Predictive Control, Economics, Real Time Optimization.

\section{INTRODUCTION}

The main goal of advanced control strategies is to operate the plants as close as possible to an economically optimal operation point, while ensuring stability. In the process industries, this objective is achieved by means of a hierarchical control structure (Qin and Badgwell, 2003): at the top of this structure, an economic scheduler and planner decides what, when and how much the plant has to produce, taking into account information from the market and from the plant. The output of this layer are production goals, prices, economic cost functions and constraints which are sent to a Real Time Optimizer (RTO). The RTO is a model-based system, operated in closed loop. It implements the economic decision in real time, performing a static optimization, and providing setpoints to the advanced control

\footnotetext{
Investigador del CONICET.

2 Becario CONICET.

3 Profesor Titular, Universidad de Sevilla.
}

level. It employs a stationary complex model of the plant and for this reason it works on a timescale of hours or day. The setpoints calculated by the RTO are sent to the advanced control level, where an advanced control strategy - usually model predictive control (MPC) (Mayne et al., 2000; Rawlings and Mayne, 2009) - calculates the optimal control action to be injcted to the plant, in order to regulate it as close as possible to the setpoint, taking into account a dynamic model of the plant, constraints, and stability requirements.

Generally, the MPC controllers are designed as setpoint-tracking controllers: the goal of the usually quadratic cost function is to drive the system as fast as possible to the desired economic setpoint. Moreover, the model used for prediction is generally a linear model: hence, the economic setpoint calculated by RTO may be inconsistent or unreachable for the dynamic layer (Kadam and Marquardt, 2007). A way to avoid this problem is to add a new optimization level in between 
of RTO and MPC, referred as the steady state target optimizer (SSTO). The SSTO calculates an admissible steady state for the linear system, solving a linear or quadratic programming and taking into account information from the RTO (Muske, 1997; Marchetti et al., 2014). In (Limon et al., 2008; Ferramosca et al., 2009, 2011) an MPC that integrates the SSTO into the same MPC layer, is presented. This controller ensures that under any change of the economic setpoint, the closed-loop system maintains the feasibility of the controller and ensures local optimality. Similar strategies are also presented in (Gonzalez and Odloak, 2009).

Recently, with the aim to add economics into the own MPC control layer, one-layer MPC controllers have been proposed. These controllers integrate the RTO economic cost function as a stationary extra cost to be added to the dynamic setpoint-tracking cost function (Zanin et al., 2002). This converts the economic objective into a process control objective, but on the other hand adds notable complexity to the optimization problem to be solved, due to the high nonlinearity of the economic cost function. Recently, a new one-layer MPC has been presented (Alamo et al., 2014), which by means of a suboptimal algorithm and the use of the gradient of the economic cost, makes the MPC optimization problem solvable with just a QP, thus reducing considerably the computational complexity.

The above mentioned controllers are designed to ensure asymptotic tracking of the economic setpoint, without taking into account the issue of transient economic costs (Angeli et al., 2012). This way to operate is practically optimal when the setpoint does not change with respect to the dynamic of the system. However, in some industrial applications, the economic cost in the transient is more significant than the cost at the steady state. This happens when the economic criterion is subject to frequent changes. Hence, it becomes very important to optimize the cost of the entire trajectory, not only at the steady state. All the above motivated in the last years the interest in Economic MPC, which considers the nonlinear economic cost of the RTO, as the stage cost for the dynamic regulation problem (Rawlings et al., 2012; Diehl et al., 2011; Amrit, 2011; Ferramosca et al., 2010, 2014).

The aim of this work is to compare different approaches to economic optimality in model predictive control, focusing in particular on the fulfillment of economic objectives, in the stationary as well as in the transient regime, and on the computation requiered to solved the MPC problem. This comparison is done by applying these controllers to a nonlinear simulator of a polymerization reactor.

\section{PROBLEM STATEMENT}

Consider a system described by a discrete-time linear time-invariant model

$$
\begin{aligned}
x^{+} & =A x+B u \\
y & =C x
\end{aligned}
$$

where $x \in \mathbb{R}^{n}$ is the system state, $u \in \mathbb{R}^{m}$ is the current control vector, $x^{+}$is the successor state, $y \in \mathbb{R}^{p}$ are the system output. The solution of this system for a given sequence of control inputs $\mathbf{u}$ and initial state $x$ is denoted as $x(j)=$ $\phi(j ; x, \mathbf{u}), j \in \mathbb{I}_{\geq 0}$, where $x=\phi(0 ; x, \mathbf{u})$. The state of the system and the control input applied at sampling time $k$ are denoted as $x(k)$ and $u(k)$ respectively.

The system is subject to hard constraints on state and input:

$$
x(k) \in X, \quad u(k) \in U
$$

for all $k \geq 0$, where $X \subset \mathbb{R}^{n}$ and $U \subset \mathbb{R}^{m}$ are compact sets.

It is assumed that the following assumption holds.

Assumption 1. The pair (A,B) is controllable and the state is measured at each sampling time.

The steady state and input of the plant $\left(x_{s}, u_{s}\right)$ are such that (1) is fulfilled, i.e. $x_{s}=A x_{s}+B u_{s}$.

We define the set of admissible equilibrium states as

$$
\begin{aligned}
& \mathcal{Z}_{s}=\{(x, u) \in \delta(X \times U) \mid x=A x+B u\} \\
& \mathcal{X}_{s}=\left\{x \in X \mid \exists u \in U \text { such that }(x, u) \in \mathcal{Z}_{s}\right\}(5) \\
& \mathcal{Y}_{s}=\left\{y=C x \mid x \in \mathcal{X}_{s}\right\}
\end{aligned}
$$

where $\delta \in(0,1)$ is a constant arbitrarily close to 1. Notice that $\mathcal{X}_{s}$ is the projection of $\mathcal{Z}_{s}$ onto $X$.

Definition 1. The economic criterion is given by the function

$$
f_{\text {eco }}(x, u, p)
$$

where $x$ and $u$ are the state and the input of the system, and $p$ is a vector of parameters which takes into account prices, costs, production goals, etc.

Function (7) represents the economic criterion to be optimized, and it may change according to the market, the plant scheduling, or the data reconciliation tasks.

The optimal operation point that stabilizes the plant, is the steady state provided by the RTO, and is defined as: 
Definition 2. The optimal steady state, input and output, $\left(x_{s}, u_{s}, y_{s}\right)$, satisfy

$$
\begin{gathered}
\left(x_{s}, u_{s}, y_{s}\right)=\arg \min _{x, u, y} f_{\text {eco }}(x, u, p) \\
\text { s.t. } x=A x+B u, y=C x, \\
x \in X, \quad u \in U
\end{gathered}
$$

and is assumed to be unique.

Remark 1. Notice that the optimal setpoint depends on the value of $p$, that is $\left(x_{s}(p), u_{s}(p), y_{s}(p)\right)$. However, for the sake of clarity, in the rest of the paper we will use the notation $\left(x_{s}, u_{s}, y_{s}\right)$.

Remark 2. Notice that here it is considered a simplified version of the RTO problem. In general, this kind of problems may also consider other objectives (not just the economic one), like nonlinear engineering constraints which cannot be normally included in the linear constraints $x \in X, u \in U$.

Next, the four representative MPC formulations considered for the study of the optimality properties are introduced.

\subsection{MPC for tracking: $M P C T$}

In the MPC for tracking the cost function is designed as a measure of the distance of the predicted trajectory to the economic setpoint $y_{s}$. In particular the formulation that we consider here (Limon et al., 2008; Ferramosca et al., 2009, 2011) guarantees recursive feasibility for any $y_{s}$ and, if possible, convergence of the system output to the setpoint.

The cost function of the proposed MPC is given by:

$$
\begin{aligned}
V_{N}^{t}\left(x, y_{s} ; \mathbf{u}\right)= & \sum_{j=0}^{N-1}\|x(j)-x(N-1)\|_{Q}^{2}+\|u(j)-u(N-1)\|_{R}^{2} \\
& +V_{O}\left(y(N-1), y_{s}\right)
\end{aligned}
$$

where $x(j)=\phi(j ; x, \mathbf{u}), Q>0$ and $R>0$. Function $V_{O}: \mathbb{R}^{p} \rightarrow \mathbb{R}$ is the so-called offset cost function and is defined as:

Definition 3. $V_{O}\left(y, y_{s}\right)$ is a positive definite convex function such that the unique minimizer of $\min _{y \in \mathcal{Y}_{s}} V_{O}\left(y, y_{s}\right)$ is $y_{s}$.

This additional cost is necessary in order to guarantee convergence to $\left(x_{s}, u_{s}, y_{s}\right)$.

The controller is derived from the solution of the optimization problem $P_{N}\left(x, y_{s}\right)$ given by:

$$
\begin{aligned}
\min _{\mathbf{u}} & V_{N}^{t}\left(x, y_{s} ; \mathbf{u}\right) \\
\text { s.t. } & \\
& x(0)=x, \\
& x(j+1)=A x(j)+B u(j), \quad \mathbb{I}_{0: N-1}(10 \mathrm{c}) \\
& y(j)=C x(j), \quad \mathbb{I}_{0: N-1} \\
& x(j) \in X, u(j) \in U \quad \mathbb{I}_{0: N-1} \\
& x(N)=x(N-1)
\end{aligned}
$$

Remark 3. Notice that constraints (10f) defines an admissible equilibrium point, such that $x(N)=$ $x(N-1)=A x(N-1)+B u(N-1) \in \mathcal{X}_{s}$, the pair $(x(N-1), u(N-1)) \in \mathcal{Z}_{s}$, and $y(N) \in \mathcal{Y}_{s}$.

Remark 4. Since the set of constraints (10b)-(10f) does not depend on $y_{s}$, the feasible region of $P_{N}\left(x, y_{s}\right)$ does not depend on $y_{s}$ either. Then there exists a region $\mathcal{X}_{N}$ such that for all $x \in \mathcal{X}_{N}$ and for all $y_{s} \in \mathcal{Y}_{s}, P_{N}\left(x, y_{s}\right)$ is feasible. $\mathcal{X}_{N}$ can be read as the set of states that can reach some target $y_{s} \in \mathcal{Y}_{s}$ in $N$ steps.

A detailed stability proof for this controller can be found in (Limon et al., 2008; Ferramosca et al., 2009).

\subsection{The one-layer $M P C: R T O+M P C$}

This controller integrate the RTO economic cost function as a stationary extra cost to be added to the dynamic setpoint-tracking cost function (Zanin et al., 2002). The cost function reads:

$$
\begin{aligned}
V_{N}^{r}(x, p ; \mathbf{u})= & \sum_{j=0}^{N-1}\|x(j)-x(N-1)\|_{Q}^{2}+\|u(j)-u(N-1)\|_{R}^{2} \\
& +f_{\text {eco }}(x(N-1), u(N-1), p)
\end{aligned}
$$

The control law is derived from the solution of the optimization problem $P_{N}^{r}(x, p)$ given by:

$$
\begin{aligned}
& \min _{\mathbf{u}} V_{N}^{r}(x, p ; \mathbf{u}) \\
& \text { s.t. }(10 \mathrm{~b})-(10 \mathrm{f})
\end{aligned}
$$

Notice that neither the cost function nor the optimization problem depends on the economic setpoint $y_{s}$. It is the own MPC controller that will determine this point by driving the system to the minimizer of the economic cost function $f_{\text {eco }}$.

\subsection{Gradient based MPC: subRTO+MPC}

The controller presented in (Alamo et al., 2014) is a low-cost formulation of the previous one. Instead of the nonlinear cost $f_{\text {eco }}$, the gradient of this function is added as extra cost to the MPC controller. Moreover, instead of injecting to the 
plant the optimal solution of the approximated problem, a solution that is the convex combination of a previously known feasible solution and the approximated one, is computed. This way, a suboptimal MPC strategy is obtained, which however ensures recursive feasibility and convergence to the optimal steady state in the economic sense, with a reduced computational cost.

The cost function to be minimized reads:

$$
\begin{aligned}
V_{N}^{s}(x, \hat{\mathbf{u}}, p ; \mathbf{u}) & =\sum_{j=0}^{N-1}\|x(j)-x(N-1)\|_{Q}^{2}+\|u(j)-u(N-1)\|_{R}^{2}(13) \\
& +\nabla f_{\operatorname{eco}(\hat{x}(N-1), \hat{u}(N-1), p)}\left[\begin{array}{l}
x(N-1)-\hat{x}(N-1) \\
u(N-1)-\hat{u}(N-1)
\end{array}\right]
\end{aligned}
$$

where $(\hat{\mathbf{u}}, \hat{x}(N-1), \hat{u}(N-1))$ at time $k$ is a previously known feasible solution, obtained using the shifted solution applied to the system at the sample time $k-1$.

The control law at time $k$ is derived from $\mathbf{u}(k)=$ $\lambda \mathbf{u}^{*}+(1-\lambda) \hat{\mathbf{u}}, \lambda \in(0,1)$, which is a convex combination of $\hat{\mathbf{u}}$ with $\mathbf{u}^{*}$, being the last one the solution of the optimization problem $P_{N}^{s}(x, p)$ :

$$
\begin{gathered}
\min _{\mathbf{u}} V_{N}^{s}(x, \hat{\mathbf{u}}, p ; \mathbf{u}) \\
\text { s.t. }(10 \mathrm{~b})-(10 \mathrm{f})
\end{gathered}
$$

A detailed stability proof for this controller can be found in (Alamo et al., 2014).

\subsection{Economic $M P C-E M P C T$}

The economic MPC considers the economic cost function as the dynamic stage cost of the MPC controller (Rawlings et al., 2012; Ferramosca et al., 2014). The cost function reads:

$$
\begin{aligned}
V_{N}^{e}\left(x, y_{s}, p ; \mathbf{u}\right) & =\sum_{j=0}^{N-1} f_{e c o}\left(x(j)-x(N-1)+x_{s}, u(j)-u(N-1)+u_{s}, p\right) \\
& +V_{O}\left(y(N-1), y_{s}\right)
\end{aligned}
$$

The control law is derived from the solution of the optimization problem $P_{N}^{e}\left(x, y_{s}, p\right)$ given by:

$$
\begin{gathered}
\min _{\mathbf{u}} V_{N}^{e}\left(x, y_{s}, p ; \mathbf{u}\right) \\
\text { s.t. }(10 \mathrm{~b})-(10 \mathrm{f})
\end{gathered}
$$

Function $V_{O}\left(y(N-1), y_{s}\right)$ is the same as in (9). Notice also that in this formulation, as in the MPCT one, we need to know the value of the economic setpoint $\left(x_{s}, u_{s}, y_{s}\right)$, which means that we need to solve the RTO problem (8) prior to the MPC problem. A detailed stability proof for this controller can be found in (Ferramosca et al., 2014).

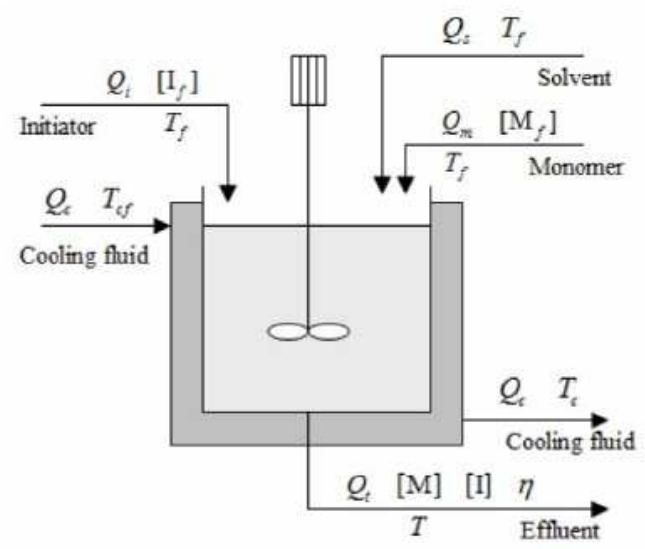

Fig. 1. Process diagram for the styrene polymerization reactor.

\section{POLYMERIZATION REACTOR - CSTR}

The four controllers have been compared in a simulation test on a nonlinear polymerization reactor system. The polymerization reactor is usually the heart of the polymer production process and its operation may be difficult as it involves exothermic reactions, unknown reaction kinetics and high viscosity (Alvarez and Odloak, 2012). Most styrene polymers are produced through batch or continuous polymerization processes. The present work considers the free-radical bulk and solution styrene polymerization in a jacketed CSTR. As shown in Fig. 1, the CSTR has three feed streams: the pure styrene monomer, the 2,2'azoisobutyronitrile (AIBN) initiator dissolved in benzene, and the pure benzene solvent. The exit stream contains polymer, un-reacted monomer, initiator, and solvent. The kinetic mechanism used for this homopolymerization process is very general and can be described by the following steps (Jaisinghani and Ray, 1977):

$I \stackrel{f_{i}, k_{d}}{\longrightarrow} 2 R$ (initiator decomposition)

$M+R \stackrel{k_{i}}{\longrightarrow} P_{i}$ (chain initiation)

$P_{n}+M \stackrel{k_{p}}{\longrightarrow} P_{n+1}$ (propagation)

$P_{n}+P_{m} \stackrel{k_{t d}}{\longrightarrow} T_{n}+T_{m}$ (termination by disproportionation)

$P_{n}+P_{m} \stackrel{k_{t c}}{\longrightarrow} T_{n+m}$ (termination by combination)

The two initiation reactions involve the decomposition of initiator I to produce radicals $R$, which react with the monomer molecules $\mathrm{M}$ to initiate new live (radical) polymer chains $P_{1}$. During the propagation step, monomer molecules $\mathrm{M}$ are added, one at a time, to the live-polymer chains $P_{n}(n \geq 1)$. The growth of the chains terminates when the propagating radicals lose their activity through any termination reaction, resulting in dead-polymer chains, $T_{n}(n \geq 1)$.

The nonlinear model that represent the dynamic of the reactor is defined by the following equations (Maner et al., 1996): 


$$
\begin{aligned}
\frac{d[I]}{d t} & =\frac{\left(Q_{i}\left[I_{f}\right]-Q_{t}[I]\right)}{V}-k_{d}[I] \\
\frac{d[M]}{d t} & =\frac{\left(Q_{m}\left[M_{f}\right]-Q_{t}[M]\right)}{V}-k_{p}[M][P] \\
\frac{d T}{d t} & =\frac{Q_{t}\left(T_{f}-T\right)}{V}+\frac{\left(-\Delta H_{r}\right)}{\rho C_{p}} k_{p}[M][P] \\
& -\frac{h A}{\rho C_{p} V}\left(T-T_{c}\right) \\
\frac{d T_{c}}{d t} & =\frac{Q_{c}\left(T_{c f}-T_{c}\right)}{V_{c}}+\frac{h A}{\rho_{c} C_{p c} V_{c}}\left(T-T_{c}\right)
\end{aligned}
$$

where

$$
\begin{aligned}
{[P] } & =\left[\frac{2 f_{i} k_{d}[I]}{k_{t}}\right]^{0.5} \\
k_{j} & =A_{j} \exp \left(\frac{-E_{j}}{T}\right), \quad j=d, p, t \\
Q_{t} & =Q_{i}+Q_{s}+Q_{m}
\end{aligned}
$$

The definition of the parameters and variables involved in the equations above can be found in (Alvarez and Odloak, 2012, Table 1 and 2). The moment equations for the dead polymer are written as follows:

$$
\begin{aligned}
\frac{d D_{0}}{d t} & =0.5 k_{t}[P]^{2}-\frac{Q_{t} D_{0}}{V} \\
\frac{d D_{1}}{d t} & =M_{m} k_{p}[M][P]-\frac{Q_{t} D_{1}}{V} \\
\frac{d D_{2}}{d t} & =5 M_{m} k_{p}[M][P]+3 M_{m} \frac{k_{p}{ }^{2}}{k_{t}}[M]^{2}-\frac{Q}{V} D_{2}
\end{aligned}
$$

$D_{0}, D_{1}$ and $D_{2}$ represent the zero, the first and the second order moment of the dead polymer, respectively.

The weight-average molecular weight is obtained as:

$$
\bar{M}_{w}=M_{m} \frac{D_{2}}{D_{1}}
$$

For online control, viscosity is commonly measured as a substitute for the average molecular weights. The following correlation is used to simulate the measurement of the viscosity (Gazi et al., 1996):

$$
\eta=0.0012 M_{w}^{0.71}
$$

\section{APPLICATION TO THE CSTR}

\subsection{Linear Model for predictions}

Here, the polymer intrinsic viscosity $\eta$ and the reactor temperature $T$ are defined as the controlled outputs. For controlling $y_{1}=\eta$ and $y_{2}=T$, the controller manipulates the initiator flow-rate $\left(u_{1}=Q_{i}\right)$ and the liquid flow rate of the cooling jacket $\left(u_{2}=Q_{c}\right)$ because of the adequate sensitivity of the process outputs to these variables. The remaining inlet flow-rates $Q_{s}$ and $Q_{m}$ are related to $Q_{i}$ by ratio control. So as to improve the performance of the controller, the ratio between the initiator flow rate $Q_{i}$ and monomer flow rate $Q_{m}$ is maintained fixed, then:

$$
Q_{m}=\frac{\bar{Q}_{m}}{\bar{Q}_{i}} Q_{i}
$$

where $\bar{Q}_{m}$ and $\bar{Q}_{i}$ are the nominal values of $Q_{m}$ and $Q_{i}$, respectively. On the other hand, the solvent volume fraction should be maintained at 0.6 to avoid the gel effect (Hidalgo and Brosilow, 1990), then a control law for the solvent flow rate is implemented as:

$$
Q_{s}=1.5 Q_{m}-Q_{i}
$$

To test the proposed controller a linear model of the styrene reactor is required. This model was obtained by subspace identification techniques. The linearization point were the simulations will start is given by: $u_{s s}=(0.030 ; 0.131)$ and $y_{s s}=$ $(3.8968 ; 323.56)$. The PRBS signal used to excite the system has an amplitude of $0.1 u_{s s}$. The identified linear model is:

$$
\begin{aligned}
& A=\left[\begin{array}{ccc}
0.9788 & 0.0292 & 0.0010 \\
-0.0006 & 0.9375 & 0.0011 \\
0.0124 & -0.0327 & 0.9569
\end{array}\right], \\
& B=\left[\begin{array}{cc}
10.2205 & -1.2333 \\
-6.6059 & -1.3983 \\
-7.1717 & 0.2481
\end{array}\right], \\
& C=\left[\begin{array}{ccc}
-0.0757 & 0.0447 & -0.1073 \\
0.6023 & -0.2749 & -0.0256
\end{array}\right]
\end{aligned}
$$

The control scheme is been equipped with a state observer and disturbance estimator of the form:

$$
\begin{aligned}
& \hat{x}^{+}=A \hat{x}+B u+L_{x}\left(C \hat{x}+\hat{d}-y_{p}\right) \\
& \hat{d}^{+}=\hat{d}+L_{d}\left(C \hat{x}+\hat{d}-y_{p}\right)
\end{aligned}
$$

where $\left(L_{x}, L_{d}\right)$ are the gain of the state observer and the disturbance estimator respectively, and $y_{p}$ is the output from the plant.

\subsection{Economic function and constraints}

Following (Alvarez and Odloak, 2012), in the RTO problem, the maximization of the production rate plus a separation cost (assumed to be directly proportional to the flow rate of the cooling jacket) is considered as the economic objective. The production rate is defined as the product of the total flowrate $Q_{t}$ and the first order moment $D_{1}$. This product represents the total weight of dead polymer produced per time unit. The economic cost function is given by

$$
f_{\text {eco }}=Q_{t} D_{1}+\left(p(1) Q_{c} T-p(2) Q_{c}\right)
$$

where $p=(p(1), p(2))$ are prices. The RTO optimization problem reads: 


$$
\begin{array}{cl}
\max _{y, u} & f_{\text {eco }}(x, u, p) \\
\text { s.t. } & \\
& x=f(x, u), \\
& y=h(x, u), \\
& y \in \mathcal{Y}, u \in \mathcal{U}
\end{array}
$$

where $\mathcal{Y}=\left\{y \mid[3 ; 310]^{\prime} \leq y \leq[5.5 ; 331]^{\prime}\right\}$ and $\mathcal{U}=\left\{y \mid[0.01 ; 0.08]^{\prime} \leq y \leq[0.07 ; 0.25]^{\prime}\right\}$.

For the linear MPC control problems, the state constraints are given by $\mathcal{X}=\left\{x \mid\|x\|_{\infty} \leq 20\right\}$.

The simulations start at the nominal operating point $\left(u_{s s}, y_{s s}\right)$. Three changes of prices have been considered: $p_{1}=(1 ; 1), p_{2}=(1.5 ; 1)$ and $p_{3}=$ $(0.1 ; 5)$.

\subsection{Economic optimality}

Figures 2, 3 and 4 show the results of the application of the four considered controllers to the nonlinear CSTR.

The first thing that we notice is how all the controllers drive the system to the economically optimal setpoint, guaranteed feasibility and ensuring stability.

As we expected, the main difference between the four evolutions is in the way they drive the system to the economic optimum.

It can be noticed in particular, that the EMPCT (solid black line), has a transient response very different from the other three controllers. This is because it optimizes the economics in the dynamic part of the MPC cost function, hence the evolution of the system under the EMPCT controller is also optimal in the transient. The other three controllers only consider economics in the stationary term of the cost function. This property of the EMPCT is of great interest, above all in those cases of frequent changes in the economics objective, or in case of cyclic economically optimal behaviours.

Another interesting aspects that we can notice is that the subRTO+MPC speeds up the convergence to the economic setpoint: this is due to the knowledge of the gradient of the economic cost function, which allows to drive the stationary part of the cost faster to the economic setpoint.

The MPCT, in the second change of the economic cost, is not able to drive the system to the economically optimal point. This fact is particularly clear in Figure 4. This is due to the fact that the $\mathrm{MPCT}$, in its stationary part of the cost (the offset cost function), only tries to minimize the distance to the setpoint, without taking into account any other information, such as an economic cost. Notice how, as a consequence, the value of $f_{\text {eco }}$ when the nonlinear system is controlled by the MPCT, is clearly not the optimal one.

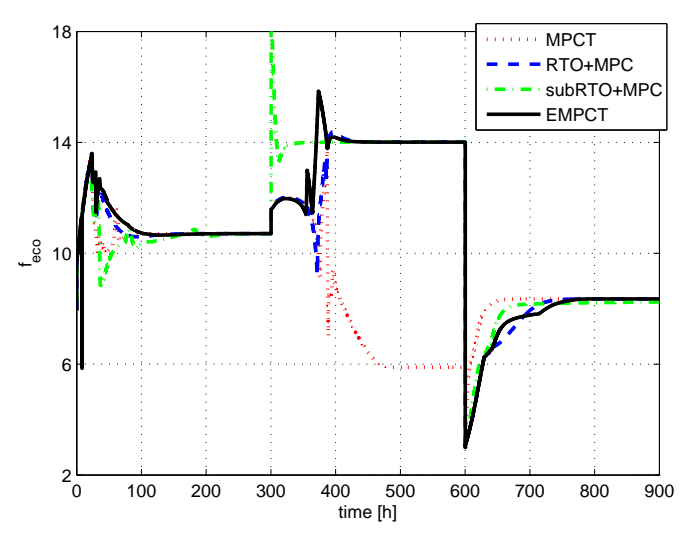

Fig. 2. Time evolution of the economics: black solid line the EMPCT, in green dash-dotted line the suboptimal $\mathrm{RTO}+\mathrm{MPC}$, in blue dashed line the RTO+MPC, and red dotted line the MPCT.
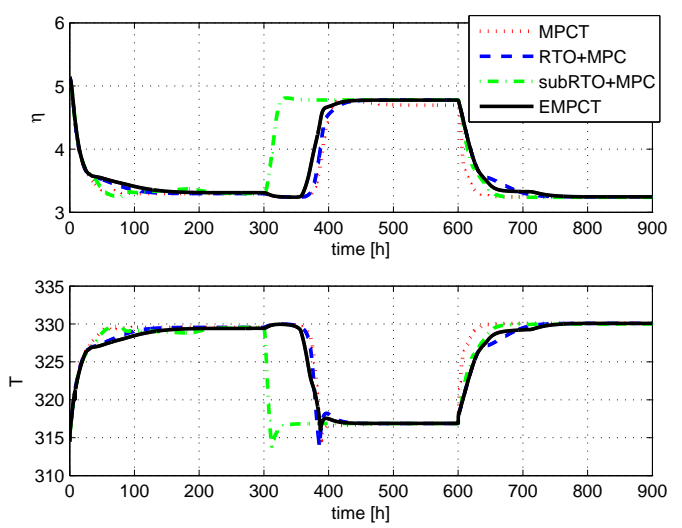

Fig. 3. Time evolution of the outputs: black solid line the EMPCT, in green dash-dotted line the suboptimal $\mathrm{RTO}+\mathrm{MPC}$, in blue dashed line the RTO+MPC, and red dotted line the MPCT.
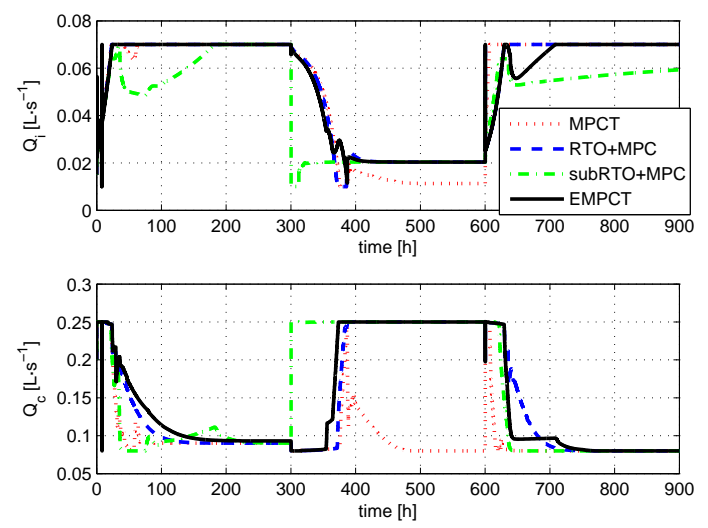

Fig. 4. Time evolution of the inputs: black solid line the EMPCT, in green dash-dotted line the suboptimal $\mathrm{RTO}+\mathrm{MPC}$, in blue dashed line the RTO+MPC, and red dotted line the MPCT.

\subsection{Computational burden}

Table 1 presents the execution times of a single MPC iteration, for the four considered controllers. The MPCT is clearly the faster algorithm since it needs to solve just a QP problem. 
Tabla 1. Execution time (in seconds)

\begin{tabular}{|c|c|c|c|c|}
\hline & Algo. & Max & Min & Average \\
\hline MPCT & QP & 0.4591 & 0.0096 & 0.0230 \\
\hline RTOMPC & SQP & 0.6880 & 0.0182 & 0.0807 \\
\hline sub-RTOMPC & QP & 0.6114 & 0.0065 & 0.0096 \\
\hline EMPCT & SQP & 0.9382 & 0.0257 & 0.1393 \\
\hline
\end{tabular}

The subRTO+MPC is also very fast in the QP solution; it's average execution time, is even lower than the MPCT one. However, it has to be underlined that here we are only considering the optimization solution time. If we also consider the calculation of the gradient, the subRTO+MPC algorithm execution time ends up to be higher. This issue however might be solved by providing an approximated gradient (such as BFGS or simular) to the controller.

The solution of the EMPC problem is clearly the one that needs more computational time, due to the high nonlinearity of the cost function.

\section{CONCLUSIONS}

In this work, economics in MPC have been studied. In particular, the economic performance of four different economic approaches have been compared.

The results have shown how each of the considered approaches is capable to ensure convergence, feasibility and stability, always fulfilling constraints. The main differences between these approaches are in the economic performance. Each of them has a different behaviour, due to its particular formulation. While setpoint tracking controllers speed up convergence to the setpoint, Economic MPC also provide economically optimal transient trajectories.

The computational burden is of course also strictly connected with the proper formulation of each controller, with RTO+MPC and EMPCT being the most computational expensive, due to the nonlinearities in their formulations. As for implementation, it should be noticed that either the MPCT and the EMPCT needs to know the economic setpoint (see (9) and (15)), hence an $a$ priori RTO problem is needed to be solved. On the other hand, RTO+MPC and subRTO+MPC are able to drive the system to the economically optimal setpoint by themselves.

It is clear from this study that, when it comes to economics, MPCT is the less interesting controller. EMPCT ensures economic optimality, in particular in the transient fase. $\mathrm{RTO}+\mathrm{MPC}$ and subRTO+MPC are able to ensure stationary economics optimality, but above all they make the presence of an RTO layer unnecessary.

\section{REFERENCIAS}

Alamo, T., A. Ferramosca, A. H. González, D. Limon and D. Odloak (2014). A gradientbased strategy for the one-layer RTO+MPC controller. Journal of Process Control 24(4), 435-447.

Alvarez, L. A. and D. Odloak (2012). Optimization and control of a continuous polymerization reactor. Brazilian Journal of Chemical Engineering 29(4), 807-820.

Amrit, Rishi (2011). Optimizing Process Economics in Model Predictive Control. PhD thesis. University of Wisconsin, Madison.

Angeli, D., R. Amrit and J. B. Rawlings (2012). On average performance and stability of economic model predictive control. IEEE Trans. on Automatic Control 57(7), 1615-1626.

Diehl, M., R. Amrit and J. B. Rawlings (2011). A Lyapunov function for Economic Optimizing Model Predictive Control. IEEE Transactions on Automatic Control 56(3), $703-707$.

Ferramosca, A., D. Limon and E. F. Camacho (2014). Economic MPC for a Changing Economic Criterion for Linear Systems. IEEE Transactions on Automatic Control 59(10), - .

Ferramosca, A., D. Limon, I. Alvarado, T. Alamo and E. F. Camacho (2009). MPC for tracking with optimal closed-loop performance. Automatica 45(8), 1975-1978.

Ferramosca, A., D. Limon, I. Alvarado, T. Alamo, F. Castaño and E. F. Camacho (2011). Optimal MPC for tracking of constrained linear systems. Int. J. of Systems Science 42(8), 1265-1276.

Ferramosca, A., J. B. Rawlings, D. Limon and E. F. Camacho (2010). Economic MPC for a changing economic criterion. In: Proceedings of 49th IEEE Conference on Decision and Control, CDC 2010. Atlanta, GE, USA.

Gazi, E., W. Seider and L. Ungar (1996). Verification of controllers in the presence of uncertainty: Application to styrene polymerization.. Ind. Eng. Chem. 35, 2277-2287.

Gonzalez, A. H. and D. Odloak (2009). A stable MPC with zone control. Journal of Process Control 19(1), 110-122.

Hidalgo, P. and C. Brosilow (1990). Nonlinear model predictive control of styrene polymerization at unstable operating points.. Comp. Chem. Eng. 14, 481-494.

Jaisinghani, R. and W. Ray (1977). On the dynamic behaviour of a class of homogeneous continuous stirred tank polymerization reactor. Chem. Eng. Sci. 32, 811-825.

Kadam, J. V. and W. Marquardt (2007). Integration of economical optimization and control for intentionally transient process operation. In: International Workshop on Assessment and Future Direction of Nonlinear Model Predictive Control (R. Findeisen, F. Allgöwer and L. T. Biegler, Eds.). pp. 419-434. Springer. 
Limon, D., I. Alvarado, T. Alamo and E. F. Camacho (2008). MPC for tracking of piecewise constant references for constrained linear systems. Automatica 44(9), 2382-2387.

Maner, B., F. Doyle III, B. Ogunnaike and R. Pearson (1996). Nonlinear model predictive control of a simulated multivariable polymerization reactor using second-order volterra models. Automatica 32, 1285 - 1301.

Marchetti, A. G., A .Ferramosca and A. H. González (2014). Steady-state target optimization designs for integrating realtimeoptimization and model predictive control. Journal of Process Control 24(1), 129-145.

Mayne, D. Q., J. B. Rawlings, C. V. Rao and P. O. M. Scokaert (2000). Constrained model predictive control: Stability and optimality. Automatica 36(6), 789-814.

Muske, K. (1997). Steady-state target optimization in linear model predictive control. In: Proceedings of the ACC.

Qin, S. J. and T. A. Badgwell (2003). A survey of industrial model predictive control technology. Control Engineering Practice 11(7), 733-764.

Rawlings, J. B. and D. Q. Mayne (2009). Model Predictive Control: Theory and Design. 1st ed.. Nob-Hill Publishing.

Rawlings, J. B., D. Angeli and C. N. Bates (2012). Fundamentals of Economic Model Predictive Control. In: Proceedings of the 51st IEEE Conference on Decision and Control (CDC). pp. 3851-3861.

Zanin, A. C., M. Tvrzska de Gouva and D. Odloak (2002). Integrating real time optimization into the model predictive controller of the FCC system. Control Engineering Practice 10(8), 819-831. 